\title{
Respuestas cardiorrespiratorias y metabólicas al ejercicio realizado sobre una plataforma de disipación de aire
}

\author{
José Luis Maté Muñoz ${ }^{a, *}$, Alessandra Moreira Reis ${ }^{b}$, Cristina López de Subijana ${ }^{b}$, \\ Bárbara Rodríguez Rogríguez ${ }^{a}$, Alberto Sacristán Rubio ${ }^{c}$, Pedro Ruiz Solano ${ }^{a}$ \\ y Manuel Vicente Garnacho Castaño ${ }^{a}$
}

a Departamento de Ciencias de la Actividad Física y el Deporte, Universidad Alfonso X el Sabio, Villanueva de la Cañada, Madrid, España

b Departamento de Ciencias Sociales de la Actividad Deportiva y Ocio, Instituto Nacional de Educación Física, Madrid, España

c Clínica de Fisioterapia y Rehabilitación Marta Alegre, Madrid, España

Recibido el 22 de julio de 2013; aceptado el 3 de diciembre de 2013

Disponible en Internet el 31 de marzo de 2014

\section{PALABRAS CLAVE \\ Actividad física; \\ Personas sanas; \\ Frecuencia cardíaca; \\ Lactato; \\ Percepción \\ de esfuerzo}

\begin{abstract}
Resumen
Introducción: El objetivo del estudio fue analizar el efecto del ejercicio realizado sobre una plataforma de disipación de aire comparado con el mismo ejercicio en suelo en un grupo de mujeres sanas.

Material y métodos: En un estudio cuasi-experimental 14 mujeres sanas de entre 20 y 25 años realizaron un mismo ejercicio en 2 condiciones diferentes separados entre sí por una semana; uno fue sobre una plataforma de disipación de aire y otro sobre el suelo. Durante las 2 pruebas se recolectaron los datos del intercambio respiratorio por un analizador de gases de circuito abierto. La frecuencia cardíaca (FC) fue registrada mediante telemetría. Muestras de sangre capilar $(5 \mu \mathrm{l})$ fueron extraídas cada $10 \mathrm{~min}$ de ejercicio utilizando un analizador de lactato portátil. La percepción subjetiva del esfuerzo (RPE) fue registrada cada $10 \mathrm{~min}$ de ejercicio mediante la escala de Borg.

Resultados: Hubo diferencias significativas en las variables de FC media (plataforma: 173,1 $\pm 13,6 \mathrm{lpm}$; suelo: $166,7 \pm 14,1 \mathrm{lpm} ; \mathrm{z}=1,9 ; \mathrm{p}<0,05$ ), ventilación (VE) media (plataforma: $58 \pm 8,6 \mathrm{l} / \mathrm{min}$; suelo: $54,4 \pm 12,4 \mathrm{l} / \mathrm{min} ; \mathrm{z}=1,7 ; \mathrm{p}<0,05$ ), lactato en el minuto 20 (plataforma: $6,9 \pm 2,4 \mathrm{mmol} / \mathrm{l}$; suelo: $4,4 \pm 1,9 \mathrm{mmol} / \mathrm{l} ; \mathrm{z}=2,9 ; \mathrm{p}<0,01$ ), en el minuto 30 (plataforma $7,1 \pm 2,6 \mathrm{mmol} / \mathrm{l}$; suelo: $5,0 \pm 2,3 \mathrm{mmol} / \mathrm{l} ; \mathrm{z}=2,4 ; \mathrm{p}<0,01$ ) y en el minuto 40 (plataforma: $5,0 \pm 1,9 \mathrm{mmol} / \mathrm{l}$; suelo: $3,6 \pm 1,6 \mathrm{mmol} / \mathrm{l} ; \mathrm{z}=1,9 ; \mathrm{p}<0,05)$, sin encontrar diferencias significativas en el RPE durante toda la prueba.

Conclusiones: La intensidad del ejercicio realizado sobre la plataforma de disipación de aire ante una misma sesión de ejercicio es mayor que la realizada sobre el suelo, teniendo unos valores similares en la percepción de esfuerzo.

(c) 2013 Consell Català de l'Esport. Generalitat de Catalunya. Publicado por Elsevier España, S.L. Todos los derechos reservados.
\end{abstract}

\footnotetext{
* Autor para correspondencia.

Correo electrónico: jmatmuo@uax.es (J.L. Maté Muñoz).
}

1886-6581/\$ - see front matter @ 2013 Consell Català de l’Esport. Generalitat de Catalunya. Publicado por Elsevier España, S.L. Todos los derechos reservados. http://dx.doi.org/10.1016/j.apunts.2013.12.001 


\section{KEYWORDS}

Physical activity; Healthy people; Heart rate; Lactate; Perceived exertion
Cardiorespiratory and metabolic responses to exercise performed on an air dissipation platform

\begin{abstract}
Introduction: The aim of the study was to analyze the effect of exercise performed on an air dissipation platform compared to the same exercise on the floor in a group of healthy women. Material and methods: In a quasi-experimental study 14 healthy women between 20 and 25 years performed exercises under two different conditions separated by one week, one was on an air dissipation platform and another on the floor. Respiratory exchange data were collected during the two tests using an open-circuit gas analyzer. Heart rate (HR) was recorded via telemetry. Capillary blood samples $(5 \mu \mathrm{l}$ ) were taken every 10 minutes during the exercises using a portable lactate analyzer. The rated perceived exertion (RPE) was recorded every 10 minutes during the exercises using the Borg scale.

Results: There were significant differences in mean HR variables (platform: $173.1 \pm 13.6 \mathrm{bpm}$; floor: $166.7 \pm 14.1 \mathrm{bpm} ; \mathrm{z}=1.9 ; P<.05$ ), average ventilation (VE) (platform: $58 \pm 8.6 \mathrm{~L} / \mathrm{min}$; floor: $54.4 \pm 12.4 \mathrm{~L} / \mathrm{min} ; \mathrm{z}=1.7 ; \quad P<.05$ ), lactate in the 20th minute (platform: $6.9 \pm$ $2.4 \mathrm{mmol} / \mathrm{L}$; floor: $4.4 \pm 1.9 \mathrm{mmol} / \mathrm{L}, \mathrm{z}=2.9 ; P<.01$ ), 30th minute (platform: $7.1 \pm 2.6 \mathrm{mmol} / \mathrm{L}$; floor: $5.0 \pm 2.3 \mathrm{mmol} / \mathrm{L} ; \quad z=2.4 ; \quad P<.01$ ) and 40 th (platform: $5.0 \pm 1.9 \mathrm{mmol} / \mathrm{L}$; floor: $3.6 \pm 1,6 \mathrm{mmol} / L ; z=1.9 ; P<.05)$, with no significant differences in RPE throughout the test. Conclusions: The intensity of exercise on the air dissipation platform with the same session of exercises is greater than that performed on the floor, with similar values in the perceived exertion.

(c) 2013 Consell Català de l'Esport. Generalitat de Catalunya. Published by Elsevier España, S.L. All rights reserved.
\end{abstract}

\section{Introducción}

La inactividad física se reconoce como uno de los factores más importantes de riesgo para la salud, incrementando el número de enfermedades crónicas en todo el mundo ${ }^{1}$. La inactividad física aumenta el riesgo de obesidad, de enfermedad coronaria, de accidente cerebrovascular, de diabetes tipo 2, así como de cáncer de colon y de mama².

Los entornos físicos, económicos y sociales en los que hoy en día viven los seres humanos han cambiado rápidamente, y en particular desde mediados del siglo pasado. Los cambios en el transporte, en los medios de comunicación, en el trabajo, así como las nuevas tecnologías en el entretenimiento y el ocio, se han asociado con una reducción significativa de la actividad física ${ }^{3}$.

Por el contrario, durante mucho tiempo la actividad física regular ha sido considerada como un componente importante de un estilo de vida saludable. Para la mayoría de las personas adultas sin patologías, el American College of Sport Medicine $(\mathrm{ACSM})^{4}$ aconseja realizar un entrenamiento cardiorrespiratorio a intensidad moderada, entre el 40 y el $59 \%$ de la frecuencia cardíaca (FC) de reserva (FCR), durante un mínimo de $30 \mathrm{~min}$, 5 días o más por semana, realizando en total un mínimo de $150 \mathrm{~min}$ a la semana. 0 bien desarrollar este entrenamiento a una intensidad vigorosa, entre el 60 y el $84 \%$ de la FCR, durante un mínimo de 20 min, 3 días o más por semana, realizando un total de $75 \mathrm{~min}$ a la semana como mínimo.

En el mundo del fitness y el wellness son muchas las actividades propuestas para estimular a la población a realizar ejercicio. Muchas de estas novedosas actividades son difíciles de realizar debido a su ejecución técnica, y en muchas otras se requieren niveles altos de condición física.
Recientemente se ha diseñado un nuevo aparato para la realización de ejercicio sobre él; consiste en una plataforma de disipación de aire que es posible incorporar en clases colectivas aeróbicas. Se compone de un área de un metro de diámetro y $20 \mathrm{~cm}$ de altura que descansa sobre un elastómero que contiene aire a presión atmosférica y que a través de orificios permite la entrada y la salida de aire; gracias a esa acción del elastómero, no se permiten rebotes. Un único estudio anterior elaborado hasta el momento ha concluido que los apoyos producidos al hacer ejercicio sobre este tipo de aparatos reducen los impactos con respecto a realizarlos en el suelo, aunque los tiempos de contacto aumentan ${ }^{5}$. Además, según demuestran los autores, ante acciones motrices iguales se percibe un menor esfuerzo subjetivo sobre este aparato que sobre el suelo, permitiendo una mayor intensidad de ejercicio. Para personas sedentarias que inician un programa de entrenamiento, realizar ejercicio sobre estas plataformas podría ser una buena solución para evitar sobrecargas y lesiones iniciales, minimizando los impactos.

Por tanto, el objetivo de nuestro estudio fue analizar el efecto del ejercicio realizado sobre una plataforma de disipación de aire comparado con el mismo ejercicio en suelo en un grupo de mujeres.

\section{Material y métodos}

\section{Participantes}

Para evaluar los efectos del ejercicio sobre la plataforma de disipación de aire se realizó un estudio cuasi-experimental en el que un grupo de mujeres realizaron un mismo ejercicio 
en 2 condiciones diferentes: uno sobre plataforma de disipación de aire y otro sobre el suelo.

Participaron 14 mujeres sanas estudiantes de Ciencias de la Actividad Física y el Deporte de la Universidad AlfonsoX el Sabio. Todas ellas realizaban actividad física ligera o moderada un máximo de 2 veces por semana. Se excluyeron mujeres entrenadas o deportistas que realizaran actividad física intensa más de 2 días por semana. Antes del estudio se les informó de todas las pruebas a realizar, y todas ellas firmaron el consentimiento de aceptación. El estudio fue aprobado por el comité ético del Departamento de Ciencias de la Actividad Física y del Deporte de la Universidad Alfonso X el Sabio, siguiendo las directrices éticas de la Declaración de Helsinki.

\section{Prueba de ejercicio}

Todas las pruebas de ejercicio se llevaron a cabo en el laboratorio de Fisiología del Ejercicio de la Universidad AlfonsoX el Sabio. Cada sujeto realizó 2 sesiones de ejercicio separadas entre sí por una semana y no realizaron ningún trabajo físico intenso durante las $48 \mathrm{~h}$ previas a dichas pruebas. Además, durante las $2 \mathrm{~h}$ previas a los test no comieron, ni fumaron ni ingirieron bebidas con cafeína; solo podían beber agua. Ambas pruebas se realizaron a la misma hora del día. Una semana antes de la primera sesión de ejercicio, para evitar episodios cardiovasculares y excluir a las participantes con alguna alteración cardiorrespiratoria, se les practicó un electrocardiograma en reposo y en ejercicio junto con una prueba de esfuerzo incremental en rampa hasta la extenuación en un tapiz rodante (TechnoGym, Runrace $1400 \mathrm{HC}$, Forlí, Italia) con una velocidad inicial de $5 \mathrm{~km} / \mathrm{h}$ y $1 \%$ de pendiente y un aumento de la carga de $0,5 \mathrm{~km} / \mathrm{h}$ cada $30 \mathrm{~s}$.

\section{Ejercicio físico}

Antes del ejercicio se extrajo una muestra de lactato en reposo $(5 \mu \mathrm{l})$ medido con un analizador portátil (Lactate Pro LT-1710, Arkray Factory Inc., KDK Corporation, Siga, Japón). Se realizó un calentamiento general estandarizado para todas las mujeres. El calentamiento de activación del organismo consistió en una carrera suave de $5 \mathrm{~min}$ en el tapiz rodante con una velocidad inicial de $5 \mathrm{~km} / \mathrm{h}$. Después efectuaron 5 min de movilidad articular y estiramientos dinámicos de las extremidades inferiores y superiores. A continuación, cada sujeto realizó una sesión de 40 min de ejercicio individual sobre una plataforma de disipación de aire imitando los movimientos a realizar de una monitora al ritmo de la música. Para controlar que fuese siempre el mismo ejercicio y en las mismas condiciones, se grabó una semana antes una clase tipo de ejercicio sobre plataforma de disipación de aire. Esta misma sesión fue reproducida en una pantalla grande a cada participante en el estudio. Se les dijo que imitasen los movimientos de la monitora manteniendo el mismo ritmo en todo momento, apoyándose en la música para conseguirlo. Los movimientos que realizaron no fueron difíciles de reproducir, no necesitando familiarización alguna. Este ejercicio se basó en movimientos globales y multiarticulares en los que participaban los grandes grupos musculares, incluyendo saltos, movimientos de brazos y pies, flexiones de tronco, etc. Durante
Tabla 1 Media de edad, peso, altura e índice de masa corporal (IMC)

\begin{tabular}{lll}
\hline Variables & Media & Desviación estándar \\
\hline Participantes & 14 mujeres & \\
Edad (años) & 22,8 & 1,3 \\
Peso $(\mathrm{kg})$ & 57,9 & 6,6 \\
Talla $(\mathrm{cm})$ & 162,3 & 0,04 \\
IMC $\left(\mathrm{kg} / \mathrm{m}^{2}\right)$ & 22,0 & 2,6 \\
\hline
\end{tabular}

toda la prueba, los datos del intercambio respiratorio fueron recogidos continuamente por un analizador de gases de circuito abierto ( $\mathrm{V}_{\max }$ spectra 29C, Sensormedics Corp., Yorba Linda, California, EE. UU.) y la FC fue monitorizada mediante registro telemétrico (RS-800CX, Polar Electro OY; Kempele, Finlandia). Muestras de sangre capilar del pulpejo del dedo índice $(5 \mu \mathrm{l})$ fueron extraídas cada 10 min de ejercicio utilizando el analizador de lactato portátil descrito anteriormente. Además, se obtuvo la percepción subjetiva del esfuerzo (RPE) cada $10 \mathrm{~min}$ de ejercicio mediante la escala de Borg ${ }^{6}$. Una semana después, cada participante realizó exactamente del mismo modo idéntica sesión de ejercicio, pero esta vez sobre el suelo. Las variables fisiológicas obtenidas fueron las medias del consumo de oxígeno $\left(\mathrm{VO}_{2}\right)$, el volumen de dióxido de carbono $\left(\mathrm{VCO}_{2}\right)$, la tasa de intercambio respiratorio (RER), la ventilación (VE), el equivalente ventilatorio de oxígeno $\left(\mathrm{VE} / \mathrm{VO}_{2}\right)$, el equivalente ventilatorio de dióxido de carbono $\left(\mathrm{VE} / \mathrm{VCO} \mathrm{CO}_{2}\right)$, el gasto energético (GE) y la FC durante los 40 min de ejercicio. $\mathrm{El}$ GE fue calculado en la prueba a carga constante mediante la fórmula $\mathrm{VO}_{2}(\mathrm{l} / \mathrm{min}) \times$ equivalente calórico del cociente respiratorio.

\section{Análisis estadístico}

Para ver el efecto del mismo ejercicio sobre plataforma de disipación de aire y sobre suelo, se aplicó el test no paramétrico de Wilcoxon para muestras relacionadas. Todos los datos se expresaron en media $(M)$ y desviación estándar (DE). El nivel de significación estadística establecido fue de $\mathrm{p}<0,05$. Para todas las pruebas se usó el programa informático SSPS versión 18.0 (SPSS, Chicago, Illinois, EE. UU.).

\section{Resultados}

Los valores descriptivos de las 14 mujeres fueron: edad: $22,79 \pm 1,25$ años; peso: $57,88 \pm 6,62 \mathrm{~kg}$; altura: $162,29 \pm 0,04 \mathrm{~cm}$; índice de masa corporal (IMC): $22 \pm 2,61 \mathrm{~kg} / \mathrm{m}^{2}$ (tabla 1 ). Los resultados del test incremental en tapiz rodante de las diferentes variables pico se detallan en la tabla 2.

En la tabla 3 se muestran los resultados de las variables cardiorrespiratorias durante el ejercicio sobre la plataforma y sobre suelo. Aparecieron diferencias significativas en la FC media alcanzada en ambos esfuerzos $(z=1,9 ; p<0,05)$. La FC en la clase con plataforma fue superior (173,1 $\pm 13,6$ lpm) a la de la clase en el suelo $(166,7 \pm 14,1$ lpm). Así mismo, la VE media durante la prueba fue significativamente superior en el ejercicio sobre plataforma $(58 \pm 8,6 \mathrm{l} / \mathrm{min})$ que sobre suelo $(54,4 \pm 12,4 \mathrm{l} / \mathrm{min}) \quad(z=1,7 ; p<0,05)$. Como 
Tabla 2 Test incremental en tapiz rodante

\begin{tabular}{lcc}
\hline Variables & Media & Desviación estándar \\
\hline $\mathrm{FC}_{\text {pico }}(\mathrm{lpm})$ & 187 & 7,8 \\
$\mathrm{VO}_{2 \text { pico }}(\mathrm{ml} / \mathrm{kg} / \mathrm{min})$ & 39,1 & 6,0 \\
$\mathrm{VO}_{2}$ a VT & 23,4 & 6,1 \\
$\%_{1} \mathrm{VO}_{2 \text { pico }}$ a VT & 59,5 & 11,6 \\
$\mathrm{VE}_{\text {pico }}(\mathrm{l} / \mathrm{m})$ & 81,3 & 16,5 \\
$\mathrm{RER}_{\text {pico }}$ & 1,23 & 0,8 \\
$\mathrm{VE} / \mathrm{VO}_{\text {pico }}$ & 37,1 & 5,3 \\
$\mathrm{VE} / \mathrm{VCO}_{\text {2pico }}$ & 32,7 & 2,3 \\
$\mathrm{RPE}$ & 14 & 1,6 \\
\hline
\end{tabular}

$\mathrm{FC}_{\text {pico: }}$ frecuencia cardíaca pico; $\mathrm{RER}_{\text {pico }}$ : tasa de intercambio respiratorio pico; $\mathrm{RPE}_{\text {final }}$ : percepción subjetiva de esfuerzo final; VE/ $\mathrm{VCO}_{2 \text { pico: }}$ equivalente de dióxido de carbono pico; $\mathrm{VE} / \mathrm{VO}_{2 \text { pico }}$ : equivalente de oxígeno pico; $\mathrm{VE}_{\text {pico }}$ : ventilación pico; $\mathrm{VO}_{2}$ a $\mathrm{VT}_{1}$ : consumo de oxígeno a umbral ventilatorio 1: $\mathrm{VO}_{2 \text { pico }}$ : consumo de oxígeno pico; $\% \mathrm{VO}_{2 \text { pico }}$ a $\mathrm{VT}_{1}$ : porcentaje del $\mathrm{VO}_{2}$ a $\mathrm{VT}_{1}$ con respecto del pico.

consecuencia de ello, también se encontraron diferencias significativas en las $\mathrm{VE} / \mathrm{VO}_{2}$ y VE $/ \mathrm{VCO}_{2}(z=2,6 ; p<0,01$, y $z=3,3 ; p<0,01$, respectivamente). En ambos casos los valores en el ejercicio con plataforma fueron superiores. No se encontraron diferencias significativas en el resto de los valores de $\mathrm{VO}_{2 \max }, \mathrm{VCO}_{2}$, RER y GE.

En la tabla 4 se muestran los resultados de las concentraciones de lactato medidas en el reposo y cada $10 \mathrm{~min}$ de prueba, siendo estas más elevadas en la sesión de ejercicio realizada sobre la plataforma que sobre suelo. Los valores registrados tras 20 y $30 \mathrm{~min}$ de ejercicios en la plataforma son cercanos a $7 \mathrm{mmol} / \mathrm{l}(6,9 \pm 2,4 \mathrm{mmol} / \mathrm{l}$ para $20 \mathrm{~min}$ y $7,1 \pm 2,6 \mathrm{mmol} / \mathrm{l}$ para $30 \mathrm{~min})$. Estos valores fueron superiores significativamente $(z=2,9 ; p<0,01$ para lactato a los $20 \mathrm{~min}$ y $z=2,4 ; p<0,01$ para lactato a los $30 \mathrm{~min}$ ) a los tomados en la clase estándar sobre pavimento normal $(4,4 \pm 1,9 \mathrm{mmol} / \mathrm{l}$ para $20 \mathrm{~min} y$ $5,0 \pm 2,3 \mathrm{mmol} / \mathrm{l}$ para $30 \mathrm{~min}$ ). Tras $40 \mathrm{~min}$ el lactato descendió a valores también superiores significativamente en plataforma $(5,0 \pm 1,9 \mathrm{mmol} / \mathrm{l})$ con respecto al pavimento normal $(3,6 \pm 1,6 \mathrm{mmol} / \mathrm{l})(z=1,9 ; p<0,05)$. No se encontraron diferencias significativas en el lactato en reposo ni en el registrado tras los $10 \mathrm{~min}$ de ejercicio.

En la tabla 5 se muestran los resultados de esfuerzo percibido en una escala de Borg de 6 a 20. Los valores mostrados tras 10, 20, 30 y 40 min de ambos tipos de superficie se situaron en un intervalo cercano a 10-11 y con una variabilidad muy baja, considerándose un valor moderado de esfuerzo durante los 40 min de ejercicio. No se encontraron diferencias significativas en ninguno de los parámetros de esfuerzo percibido.

\section{Discusión}

El hallazgo más importante de este estudio fue encontrar diferencias significativas en las concentraciones de lactato en las últimas 3 tomas (tabla 4) en 2 ejercicios idénticos (plataforma de disipación de aire y suelo), siendo la diferencia el medio sobre el cual realizaron dicho ejercicio. Esto podría ser debido a que sobre la plataforma se reduce el impacto, aumentando los tiempos de contacto del pie sobre el aparato, y ello provocaría una mayor contracción muscular, acentuando el trabajo por parte de los miembros inferiores. Estudios que comparan superficies estables con superficies que generan inestabilidad (balones suizos, balones semiesféricos) han documentado valores similares en la activación muscular, con una disminución de la fuerza máxima isométrica sobre superficies inestables ${ }^{7}$. En otro trabajo publicado por Behm et al. ${ }^{8}$, incluso los niveles de actividad electromiográfica en los músculos antagonistas y sinergistas ante ejercicios realizados en condiciones de inestabilidad se incrementaron en un 29,1 y un $30,3 \%$, respectivamente, indicando que esa mayor actividad muscular probablemente contribuya a una mayor estabilización articular, no siendo tan efectiva en el desarrollo de fuerza, aunque sí influya en un mayor trabajo muscular. Esta mayor actividad muscular podría explicar las mayores concentraciones de lactato en el ejercicio sobre plataformas de disipación de aire realizado en nuestro estudio.

Otro dato interesante de este trabajo fue encontrar diferencias significativas en la intensidad de ejercicio medida a través de la FC ante una idéntica sesión de ejercicio

Tabla 3 Valores registrados de las variables cardiorrespiratorias: media (M) y desviación estándar (DE)

\begin{tabular}{|c|c|c|c|c|c|c|}
\hline \multirow[b]{2}{*}{ Variables } & \multicolumn{2}{|c|}{ Con plataforma } & \multicolumn{2}{|l|}{ Suelo } & \multirow[t]{2}{*}{ Diferencia medias } & \multirow[t]{2}{*}{$\mathrm{p}$} \\
\hline & $M$ & DE & M & DE & & \\
\hline FC (lpm)* & 173,1 & 13,6 & 166,7 & 14,1 & 6,4 & 0,030 \\
\hline $\mathrm{VO}_{2}(\mathrm{l} / \mathrm{min})$ & 1,7 & 0,2 & 1,7 & 0,3 & 0 & 0,255 \\
\hline $\mathrm{VO}_{2}(\mathrm{ml} / \mathrm{kg} / \mathrm{min})$ & 29,1 & 2,3 & 30,0 & 4,8 & $-0,9$ & 0,165 \\
\hline $\mathrm{VCO}_{2}(\mathrm{l} / \mathrm{min})$ & 1,6 & 0,2 & 1,7 & 0,3 & $-0,1$ & 0,462 \\
\hline RER & 1,0 & 0,1 & 1,0 & 0,0 & 0 & 0,190 \\
\hline $\operatorname{VE}(\mathrm{l} / \mathrm{min})^{*}$ & 58,1 & 8,6 & 54,4 & 12,4 & 3,7 & 0,045 \\
\hline $\mathrm{VE} / \mathrm{VO}_{2}{ }^{* *}$ & 34,9 & 4,5 & 32,1 & 4,1 & 2,8 & 0,005 \\
\hline $\mathrm{VE} / \mathrm{VCO}_{2}{ }^{* *}$ & 35,3 & 3,2 & 33,2 & 3,8 & 2,1 & 0,001 \\
\hline GE (kcal) & 337,3 & 30,3 & 348,1 & 60,2 & $-10,8$ & 0,365 \\
\hline
\end{tabular}

FC: frecuencia cardíaca; GE: gasto energético; RER: tasa de intercambio respiratorio; $\mathrm{VCO}_{2}$ : volumen de dióxido de carbono; VE: ventilación; $\mathrm{VE} / \mathrm{VCO}_{2}$ : equivalente de dióxido de carbono; $\mathrm{VE} / \mathrm{VO}_{2}$ : equivalente de oxígeno; $\mathrm{VO}_{2}$ : consumo de oxígeno.

* $p<0,05$.

$p<0,01$. 
Tabla 4 Valores de las concentraciones de lactato: media (M) y desviación estándar (DE)

\begin{tabular}{|c|c|c|c|c|c|c|}
\hline \multirow[t]{2}{*}{ Variables } & \multicolumn{2}{|c|}{ Con plataforma } & \multicolumn{2}{|c|}{ Suelo } & \multirow[t]{2}{*}{ Diferencia medias } & \multirow[t]{2}{*}{$\mathrm{p}$} \\
\hline & M & DE & M & $\mathrm{DE}$ & & \\
\hline Lactato, reposo $\min (\mathrm{mmol} / \mathrm{l})$ & 1,4 & 0,4 & 1,5 & 0,3 & $-0,1$ & 0,266 \\
\hline Lactato, $10 \mathrm{~min}(\mathrm{mmol} / \mathrm{l})$ & 6,5 & 2,7 & 5,5 & 1,8 & 1 & 0,216 \\
\hline Lactato, $20 \mathrm{~min}(\mathrm{mmol} / \mathrm{l})^{* *}$ & 6,9 & 2,4 & 4,4 & 1,9 & 2,5 & 0,002 \\
\hline Lactato, $30 \mathrm{~min}(\mathrm{mmol} / \mathrm{l})^{* * *}$ & 7,1 & 2,6 & 5,0 & 2,3 & 2,1 & 0,008 \\
\hline Lactato, $40 \mathrm{~min}(\mathrm{mmol} / \mathrm{l})^{*}$ & 5,0 & 1,9 & 3,6 & 1,6 & 1,4 & 0,029 \\
\hline
\end{tabular}

Tabla 5 Valores registrados de la percepción subjetiva del esfuerzo (RPE): media (M) y desviación estándar (DE)

\begin{tabular}{|c|c|c|c|c|c|c|}
\hline \multirow[t]{2}{*}{ Variables } & \multicolumn{2}{|c|}{ Con plataforma } & \multicolumn{2}{|c|}{ Suelo } & \multirow[t]{2}{*}{ Diferencia medias } & \multirow[t]{2}{*}{$\mathrm{p}$} \\
\hline & $M$ & DE & M & $\mathrm{DE}$ & & \\
\hline RPE, 10 min & 10,4 & 1,0 & 10,2 & 1,5 & 0,2 & 0,282 \\
\hline RPE, $20 \mathrm{~min}$ & 10,9 & 1,2 & 11,1 & 1,5 & $-0,2$ & 0,103 \\
\hline RPE, $30 \mathrm{~min}$ & 11,8 & 1,3 & 11,2 & 1,5 & 0,6 & 0,072 \\
\hline RPE, 40 min & 11,7 & 1,8 & 11,2 & 1,7 & 0,5 & 0,122 \\
\hline
\end{tabular}

realizado sobre las 2 superficies. Mientras que sobre el suelo la FC medida durante toda la prueba fue de 166,7 lpm, en el ejercicio realizado sobre la plataforma fue de 173,1 lpm. Esta FC está incrementada en la plataforma con respecto al suelo en un $3,8 \%(6,4 \mathrm{lpm})$. Por tanto, podríamos decir que existe una respuesta cardíaca mayor al hacer ejercicio sobre este tipo de aparatos.

A pesar de documentar que en el ejercicio sobre plataforma de disipación de aire existe un mayor trabajo muscular y una respuesta cardíaca más acentuada con respecto al ejercicio en suelo, la percepción de esfuerzo subjetiva (RPE), medida a través de la escala de Borg, fue similar en ambas condiciones. Se obtuvieron en las 2 situaciones de ejercicio unos valores medios en torno a 11 sobre 20 de RPE, siendo esta intensidad del esfuerzo percibido moderada. Esto puede parecer paradójico, puesto que si un ejercicio es más intenso, es muy probable que la persona lo perciba como un esfuerzo mayor. Una explicación podría ser que la reducción de los impactos sobre esta plataforma podría hacer disminuir la RPE. Es decir, al existir un menor estrés en huesos, tendones, músculos y articulaciones debido a esa dispersión de la fuerza en los apoyos, se podría reducir la fatiga del sistema osteo-mio-articular. Además, los mecanorreceptores periféricos podrían transmitir una información atenuada del esfuerzo al cerebro. Esto es especialmente importante puesto que se pueden mantener intensidades altas de ejercicio sobre estas plataformas $\left(173,1 \mathrm{lpm}=92,6 \%\right.$ de la $F C_{\text {pico }}$ obtenida una semana antes en la prueba incremental en tapiz rodante), percibiendo un esfuerzo moderado, siendo estas intensidades más efectivas para mejorar la capacidad aeróbica y de esa manera obtener mayores beneficios cardioprotectores ${ }^{9}$. Respecto al $\mathrm{VO}_{2}$ obtenido durante las 2 sesiones de ejercicio, se observan valores similares (plataforma de disipación de aire $29,1 \mathrm{ml} / \mathrm{kg} / \mathrm{min}$; suelo $30,0 \mathrm{ml} / \mathrm{kg} / \mathrm{min}$ ). El valor de este
$\mathrm{VO}_{2}$ durante los $40 \mathrm{~min}$ de prueba con respecto al máximo obtenido en la prueba incremental fue del $74,4 \% \mathrm{VO}_{2 \text { pico }}$ en ejercicio sobre plataforma y del $76,7 \% \mathrm{VO}_{2 \text { pico }}$ en ejercicio sobre suelo, considerándose ambos ejercicios vigorosos según el $\mathrm{ACSM}^{10}$. Altos niveles de $\mathrm{VO}_{2 \max }$ se han asociado con una reducción del riesgo de enfermedad y mortalidad ${ }^{11,12}$, siendo más efectivo incrementar esta variable con ejercicio vigoroso $\left(60-84 \% \mathrm{VO}_{2 \text { máx }}\right.$ o $\left.\mathrm{FCR}\right)$ que con ejercicio moderado $\left(40-59 \% \mathrm{VO}_{2 \text { máx }} \text { O } \mathrm{FCR}\right)^{13}$.

Una limitación de nuestro estudio fue la muestra reducida de solo 14 mujeres sanas. Además, el laboratorio de Fisiología no es el entorno habitual donde se desarrollan este tipo de sesiones. Por tanto, serían necesarias nuevas investigaciones para corroborar los datos de este estudio con muestras mayores en entornos habituales, así como medir la reducción del impacto en la plataforma y la activación electromiográfica de la musculatura implicada en el ejercicio, tanto en el suelo como en la plataforma.

Por tanto, podríamos concluir que la respuesta cardíaca y el trabajo muscular al hacer ejercicio sobre la plataforma de disipación de aire son mayores comparados con una misma sesión de ejercicio sobre suelo, teniendo unos valores similares en la percepción de esfuerzo. Esta menor percepción de esfuerzo puede deberse a una disipación de los impactos, reduciendo el riesgo de lesión osteo-mio-articular. El ejercicio sobre estas plataformas de disipación de aire podría ser una herramienta eficaz para personas sedentarias o con sobrepeso u obesidad, obteniendo una reducción de la grasa corporal, mejorando su capacidad funcional sin tener una fatiga excesiva y manteniendo un programa de ejercicio en el tiempo, siendo constantes en la práctica de actividad física, aspecto que muchas veces es un problema en personas sedentarias. Además, podría disminuir el riesgo de lesión debido a una absorción mayor de impactos al apoyarse sobre la plataforma. Aun así, la probabilidad de 
sufrir complicaciones cardiovasculares es mayor a intensidades altas, por lo que personas con riesgo cardiovascular o desentrenados deberían iniciar ejercicios de intensidad moderada, pasando a entrenar a estas intensidades vigorosas una vez haya pasado un tiempo de adaptación.

\section{Conflicto de intereses}

Los autores declaran no tener ningún conflicto de intereses.

\section{Bibliografía}

1. Ezzati M, Lopez A, Rodgers A, Hoorn SV, Murray JL. Selected major risk factors and global and regional burden of disease. Lancet. 2002;360:1347-60.

2. Chatterji S, Gutlhold R, Ono T, Strong KL, Kathleen LS, Somnath C, et al. Worldwide variability in physical inactivity a 51 -country survey. Am J Prev Med. 2008;34:486-94.

3. Owen N, Healy GN, Mathews CE, Dunstan DW. Too much sitting: The population health science of sedentary behavior. Exerc Sport Sci Rev. 2010;38:105-13.

4. Garber CE, Blissmer B, Deschenes MR, Franklin BA, Lamonte MJ, Lee IM, et al. Quantity and quality of exercise for developing and maintaining cardiorespiratory, musculoskeletal, and neuromotor fitness in apparently healthy adults: Guidance for prescribing exercice. American College of Sports Medicine Position Stand. Med Sci Sports Exerc. 2011;43:1334-59.
5. Citta F. Valoración de la sensación subjetiva del esfuerzo en mujeres menopáusicas en trabajo de marcha sobre suelo y disipación de aire [tesis doctoral]. Argentina: Universidad CAECE; 2011.

6. Borg G. Perceived exertion as an indicator of somatic stress. Scand J Rehabil Med. 1970;2:92-8.

7. Anderson K, Behm D. Maintenance of EMG activity and loss of force output instability. J Strength Cond Res. 2004;18:637-40.

8. Behm DG, Anderson K, Curnew RS. Muscle force and activation under stable and unstable conditions. J Strength Cond Res. 2002;16:416-22.

9. Swain DP, Franklin BA. Comparison of cardio protective benefits of vigorous versus moderate intensity aerobic exercise. Am J of Cardiol. 2006;97:141-7.

10. Franklin BA, Whaley MH, Howley ET, Balady GJ, American College of Sports Medicine. ACSM's Guidelines for Exercise Testing and Prescription. Philadelphia, PA: Lippincott Williams \& Wilkins; 2000. p. 143-51.

11. Blair SN, Kohl HW, Paffenbarger RS, Clark DG, Cooper KH, Gibbons LW. Physical fitness and all-cause mortality: A prospective study of healthy men and women. Amer Medical Assoc. 1989;262:2395-401.

12. Laukkanen JA, Lakka TA, Rauramaa R, Kuhanen R, Venäläinen JM, Saloren R, et al. Cardiovascular fitness as a predictor of mortality in men. Arch Intern Med. 2001;161:825-31.

13. Swain DP. Moderate or vigorous intensity exercise: Which is better for improving aerobic fitness? Prev Cardiol. 2005;8: $55-8$. 